\title{
Narrative Evolvement From Panoramic Art to Interactive Art
}

\author{
SUN Xin \\ Academy of Fine Arts, Northeast Normal University, Jilin, Changchun 130117 china \\ 153465917@qq.com
}

Keywords: Narrative,Evolvement From Panoramic Art ,Interactive Art

\begin{abstract}
For hundreds of years, the narrative art has undergone many important changes, especially since the 1990s, the narrative art meets a Renaissance. As a new mode of communication, Internet has taken up the status of mainstream media, and it urgently needs more appropriate forms to express its artistic and literary content. This paper presents various narrative techniques, from visual, literary, to multimedia in different historical periods, focusing on the artistic means which change our perception of reality. For example, the panoramic art in the late eighteenth century, the novels of James Joyce in the early twentieth century, and the experimental films in the 1940s and 1950s. Since the late eighteenth century, the diversity of narrative forms and their performance in digital media have gone through different stages. Every description of reality comes from our own definition of reality, which in turn affects our definition of reality.
\end{abstract}

\section{Panoramic Art}

In 1787, the Irish painter Robert Barker used the "panoramic" perspective in his work for the first time. The first group of panoramic paintings appeared in Britain, France, Germany, and the United States one after another, bringing changes to the narrative and media art itself. At that time, this kind of new art form was understood as a collision of aesthetics and science. The spark of friction pointed the criticism at a discussion about the value of technological art, which was not from the invention of photography, but was emerging quietly in the period of panoramic art.

The word "panorama" was firstly used in 1792: it is a combination of PON (all) and HDROMA (see), which refers to the physical space of a wide range of views, covering a 360-degree visual space. In the panoramic image, the relationship between things has changed, and the way of narration and the form of audience acceptance have changed.

First of all, the panorama has created a multi-angle image world as an art form, where all things are equal. The details of the description are not limited to the scope defined by the pivot perspective, but are reconstructed and combined in a new visual angle. Then, people can view different parts of the work from different angles at the same time. As the audience cannot see the whole work all at once, he has to move and to raise his attention. Therefore, we believe that the panoramic art not only creates an illusion space but also more importantly enables the audience to appreciate its charm only through participation.

The basic idea of the panorama is based on the concept of history and culture; the aim is to provide an ingenious artificial image for the viewer; who can experience the feeling of being in two spaces at the same time. In this way, the scope of aesthetics starts to test the scope of human perception; this kind of unstable state of the perception goes through the pioneer art form of the whole 1920s, from impressionism, cubism, and then to the futurism. However, the instability of the perception has also become the main feature of the works of James Joyce, who is the famous stream of consciousness novelist.

\section{The stream of consciousness of James Joyce}

Stream of consciousness is a noun, which was firstly used by William James, the earliest American psychologist, in his principle of psychology (1890); the original meaning indicates that human 
consciousness is in a flow and changing, instead of fixed and methodical. .Then, psychoanalyst Freud came up with the theory of consciousness and sub consciousness. In literature, it means that novelist describes the inner thoughts of characters through association, recollection, etc., and the reaction to the external things at any time, which also can be called as the inner monologue. Joyce's stream of consciousness stream captures the incoherence and uncertainty of the characters' minds. It is messy and sprawling. He actually has a different approach in the novel Ulysses, which describes the experience, idea, and emotions of the several citizens in Dublin from eight in the morning to midnight, a total of 18 hours with Blum as the main character; it is in disorder on the one hand, but the structure is the most careful and rigorous. He describes the path of man in the eternity of space and time, reflecting the contradictions and defects of modern western civilization with a microscope of accuracy. In the whole work, it almost describes all the emotional experiences of human beings, which are acclaimed as the first novel of the stream of consciousness.

Joyce uses a nonlinear structure and a large number of metaphors that break through time constraints, which are more closely related to the expression of visual arts. His form is different, which can both stimulate the reader's cognitive potentials and make the reader compete with him. .The process of entering his work is the theme of the work itself, and the narration becomes a kind of communication with the reader's mind. The new experimental narrative strategy is difficult to interpret and highly complex in content, which is called as "a sealed book" of the history of literature; this is mainly derived from Joyce's keenness to activate the reality rather than retell the reality. When the reality appears, it is no longer fixed and rigid, but flowing and spiritual. The characters, the environment and the plot of his novels are also like this. Finnegan's Wake, in particular, almost reaches the acme, in which the objective and linear descriptions are repeated in a large amount, almost reached the acme. The novel continues to create new narrative networks, where the relationship is constantly changing and new connections are constantly emerging. Reading develops in a "network" way for the first time. It changes the an unprecedented "network" approach. This changes traditional narrative logic. In the complex narrative, the traditional causal relationship and linear narrative progress have been discarded.

In Ffinnegan's Wake, Joyce attempts to encapsulate the entire history of humanity through his dreams, and at the same time, Joyce takes his stream-of-consciousness and dream-like style to the extreme. In fact, this work has a profound effect on the underground films and experimental films of the 1940s and the 1950s. Trying to find the different time and spatial organization forms from linear narration became the main feature of the experimental film of Maya Delen. Her work is composed of one dream after another, which is the interweaving and intermingling of the inner and outer, and the reality and the dream. Her first and most prestigious work is Meshes of the Afternoon, 1943. The play is considered as a precursor to the American pioneering film. She attributes the challenge to the camera objectivity; she does not focus on the objective external world any more, but will move the creation center to the inner world: the person's psychology, pure and mysterious religious ceremony.

Although Joyce hoped to develop an aesthetic interactive art in the literary field, the medium of literature was not able to give full play to this aesthetic effect. As Joyce predicted, the film is superior to fiction in terms of expressing the emotional aspects of human beings, but his ability to express imaginary space with nonlinear descriptions is limited. However, his philosophy realizes perfectness in aesthetic sense through the digital media platform of computers.

\section{Interactive art}

In 1989, there were too many political and artistic events. In many ways, 1989 was a watershed. With the fall of the Berlin wall and the reintegration of the political landscape in Eastern Europe, it changed the political landscape that had already formed. The world was not divided into two opposing camps. A new world order emerges, conflicts and paradoxes self-reorganize, and the center of power is transferred. In this year, interactive art came to the fore. It seems that all the important interactive artists agree in Joyce's role in this new art form; such artists as Ken Feingold, Shao Zhifei, Graham Webb, and Lynn Hersherman began to create their first writing the first interactive installation works, in which Joyce's narrative strategy has achieved a perfect aesthetic sense. These 
works try new approaches, and the interaction is not only a positive interpretation, but also the realization of the imagination and the persistence separating from words. Interactive art reshuffles the social status of art categories.

The new aesthetic choices of restructuring the changed world order enables people to define a brand new and transformed social space. This has more far-reaching significance, for in the late 20th century, new media not only accelerated the process of globalization, but also fundamentally questioned the nationalism dividing cultural space and territory. .The map here is both concrete and abstract. It provides a new evaluation space where the traditional form of art expression has been completely discarded. The text and logos replace the world of matter, and the signs here are not linear, but in a network with constant extension. Such a territory structure is common in interactive art works. Shao Zhifei, an Australian artist, creates his own digital space in this way. In his space, the characters move in the space of sight, text and hearing, no longer using the vertical distribution structure, but organized like hypertext. In the process, the viewer is free to wander inside and outside the works. At this time, he is no longer a mere "blind person" or an observer in the image, because the "inside" and the "outside" are happening at the same time. The works itself is not just imagined, but moved through the personal experience. This seems similar to the early panoramic view of art and concept; actually, the aesthetic purpose of interactive art is not to make the abstract concept visualized, but to start a new experience, organizing reality in an abstract way and reflecting the reality to individual.

Interactive art adopts the method of recombining poetry to combine images, words, languages, sounds and movements together, creating a variety of new ways to form a new network space order. It can lead the user to navigate in the realm of meaning, where there is no order of time and only an instantaneous hierarchy. This method requires people to believe that they can deal with the current huge amount of cultural symbols and to have a new understanding of history. Such interactive art works can withstand the present comments on the requirements to discard the visual reappearing and begin a new vocabulary form, resisting the non-permanence of transferring the visual habit to move to the new space. Its visual language is both abstract and concrete. New metaphor appears in the world of nonphysical data, which allows us to override all technical decoding, though cultural decoding not included. In its network, all the elements are connected and influenced by each other. The user moves around it, like a aimless walk and free recognition. In this process, the meaning of works can only be presented and the aesthetic potential of interactive art can only be found out in detail by the user's positive efforts; this is also the first time to make the abstract intangible order get specific sensory explanation.

In interactive art, part of creations is transferred from the artist to the user. Just as the artist's behavior or the performance of body art represent the transformation of identity, the bystander of the past has become the current user. In a digital environment, the user finds him and perceives the impact of his actions. In normal social life, human beings seem to like to be in the rules, which seem to be more convenient; it also needs to follow the rules, instead of thinking about the sense of responsibility. In an automated environment, he has to focus on himself, and to realize that reality is in his control. In the interactive works, the user realizes that he and basic perceptions coexist. He seems to be in an all-around position to manipulate events, but he is also the victim of events. In this network, he's just one of many manipulators. This is especially true when interactive art works directly into the Internet.

Interactive art works show that our narrative strategy and our concept of building reality are already longing for new forms. This transformation has begun to emerge in the Victorian panoramic art and James Joyce's experimental literature. But only in the environment of computer and Internet, can interactive art fully release its aesthetic light.

During the Technological Renaissance of the 1970s, the link between the body and the machine was tested in the "artificial reality environment". Since the late 1980s, people have been able to experiment with the interaction in spirit and function in the interactive art works, connecting abstract programs with the human body. This association between psychological interaction and abstract process make reflexive communication possible within the range of digital culture. 
Interactive art works allow people to try a new understanding of the world. The world is no longer a linear cause-and-effect relationship, and there is no remote corner. People are also participants and creators. Interactive art is mobile, dynamic, and hierarchical relationships are temporary; therefore, only temporary levels are recognized. Its uniqueness rests on that it renews with its awareness in every moment.

Interactive art is no longer a personal expression or a collective expression; it is a "network" structure. Artists or artist group, their individual positions have been replaced by artistic practices which don't pursue eternity. Their temporary attributes constitute the precondition for their inherent potential changes. Interactive art works cannot be separated from participants' intervention, which can also indicate that the process of globalization requires as many as people to participate in.

\section{References}

[1] Xu Nanming (Main editor). Film Art Dictionary [Z]. Beijing: China Film Press, 1986.1.

[2] [France] Andre Bazin. What's film? [M].Cui Junyan, as Translator. Beijing: China Film Press, 1987.11

[3] [America] Paul Levinson. Digit Mcluhan [M] He Daokuan, as Translator. Beijing. Social Science Academic Press, 2001.

[4] [Britain] Alert Baldwin. Introduction of Cultural Studies [M]. Tao Dongfeng et al. as Translators. Beijing : Higher Education Press, 2004.

[5] [Sri Lanka ] Alessi Ales Erjavec. Image Age [M] Hu Julan, Zhang Yunpeng, as Translators, Changchun: Jilin People’s Press, 2003. 